\title{
Treatment of Hepatocellular Carcinoma in the Community: Disparities in Standard Therapy
}

\author{
Linda C. Harlan ${ }^{a}$ Helen M. Parsons ${ }^{b}$ Charles L. Wiggins ${ }^{c}$ \\ Jennifer L. Stevens ${ }^{d}$ Yehuda Z. Patt ${ }^{c}$ \\ aDivision of Cancer Control and Population Sciences, National Cancer Institute, Bethesda, Md., \\ ${ }^{b}$ Department of Epidemiology and Biostatistics, University of Texas Health Science Center at San Antonio, \\ San Antonio, Tex., 'University of New Mexico (UNM) Health Sciences Center, Albuquerque, N.M., \\ dInformation Management Services, Inc., Calverton, Md., USA
}

\section{Key Words}

Embolization · Insurance - Liver cirrhosis - Liver transplant · Population based

\begin{abstract}
Background and Aims: Hepatocellular carcinoma (HCC) incidence is expected to rise dramatically over the next decades because of increasing hepatitis $C$ infections and obesityrelated comorbidities. However, little information exists regarding the treatment of patients with HCC in the community setting. The purpose of this article was to characterize patterns of diagnosis, treatment, and survival for HCC in the community. Methods: We identified 946 HCC patients in the 2007 National Cancer Institute's Patterns of Care study. Chi-square analyses and multivariable regression were used to examine patient and provider factors associated with treatment and survival by stage at diagnosis. Results: Our primary findings indicate that liver transplants, embolization, or radiofrequency ablation for Barcelona Clinic Liver Cancer stage A patients were performed significantly less often for non-Hispanic blacks, Hispanics, patients in the highest income quartile, and patients with Medicaid. Patients with stage $D$ disease were less likely to receive cancer therapy if they had Medicaid insurance compared to private insurance $(p<0.001$ for all). In multivariable analyses, all-cause mortality was associated with treatment in a hospital without a residency training program (hazard ratio [HR] 1.4 [1.1,1.9]), more advanced stage (HR: 10.6 [5.7, 19.5] stage D vs. A), and lack of appropriate treatment (HR: 2.4 [1.9,3.2]). Conclusions: This is the first population-based study to evaluate therapy provided for HCC in the community. Current therapy depended on patients' HCC stage at diagnosis and other clinical and demographic factors. Overall, our study identi-
\end{abstract}


fies those least likely to receive specific therapies in a variety of health care settings and can inform strategies for promoting appropriate therapy now and as new agents are developed.

Copyright $\odot 2015$ S. Karger AG, Basel

\section{Introduction}

Although hepatocellular carcinoma (HCC) is a relatively rare cancer in the United States (2\%), the incidence is expected to continue rising over the next decades because of hepatitis C infection rates in the population $[1,2]$. While HCC is primarily associated with cirrhosis, it is increasingly being linked to obesity and the resulting metabolic syndrome and diabetes [3]. Patients presenting with localized disease are eligible for potentially curable surgical resection or liver transplant [4]. However, the vast majority of patients present with advanced HCC and will require systemic therapy [5, 6]. Until recently, the few systemic therapies available to patients with advanced HCC were minimally effective at extending survival and were highly toxic [7]. Currently, the development of molecularly targeted treatments has the greatest potential to improve the outcomes of patients with advanced HCC [8]. While information on treatment patterns and associated outcomes are available for patients treated in clinical trials or cancer center settings, little information is available for patients with HCC treated throughout the community.

Identifying factors related to receipt of recommended therapy for HCC patients in all treatment settings will guide future treatment and resource planning. Additionally, understanding patterns of care for patients with HCC can inform strategies for promoting appropriate therapy now and as new agents are developed for HCC. For our study, we used the National Cancer Institute's (NCI) annual Patterns of Care study to evaluate differences in patient and provider factors associated with Barcelona Clinic Liver Cancer (BCLC) stage $[9,10]$ at diagnosis, treatment received, and survival of a population-based sample of patients diagnosed and treated in diverse health care settings.

\section{Methods}

The NCI's Surveillance, Epidemiology, and End-Results (SEER) Program includes a set of populationbased registries that collect all incident cancers occurring in their defined geographic area, which covers about $28 \%$ of the US population [11]. Data are collected on demographics, diagnosis, tumor characteristics, treatment, and follow-up for vital status. Because much of the adjuvant therapy is provided in an outpatient setting and SEER data collection is primarily hospital based, the NCI annually conducts a more comprehensive data collection on a sample of patients diagnosed with specific cancers. Patients diagnosed in 2007 with hepatocellular cancer were selected for inclusion in the patterns of care study. Institutional review board approval was received as required by the registries. Prior to study initiation, central abstractor training was held to ensure consistency of data collection and coding. The hospital data were re-abstracted and each patient's treating physician was contacted to verify the treatment given and the Child-Pugh score. The physician was also asked whether other physicians might have provided care and those physicians were then contacted. All comorbidities present at the hospitalization for the most definitive therapy were collected and analyzed using the Charlson comorbidity score [12]. The presence of cirrhosis and/or hepatitis was based on documentation by the physician in the hospital medical records. If there was no indication of cirrhosis and/or hepatitis in the record, they were recorded as not present. Insurance at the time of treatment was also verified. If multiple insurance carriers were listed, all were recorded and categorized hierarchically into any private (including military), any Medicaid, Medicare only, and no insurance. Income was based on the median family income of the census tract where the patient resided when diagnosed with cancer. 
In 2007, patients diagnosed with HCC (International Classification of Diseases for Oncology, 3rd Edition: site code (topography): C22.0 and morphology codes: M 8170-75; 8180) and registered to one of 14 registries were identified. Because of extremely small numbers, patients were ineligible if they were less than 20 years of age. Patients who had a previous diagnosis of cancer (other than non-melanoma skin cancer) or were diagnosed simultaneously with another cancer were excluded because we could not be certain that the HCC treatment would not be influenced by these diagnoses. Patients diagnosed at autopsy or on death certificate only were also excluded. Eligible cases were stratified by registry, age, race/ethnicity, and sex and a random sample of patients was selected within strata ( $\mathrm{n=946).} \mathrm{Non-Hispanic} \mathrm{blacks,}$ Hispanics, Asian/Pacific Islanders and American Indians/Alaskan Natives were oversampled to obtain more stable estimates, but our final weighted sample is representative of the SEER population in 2007.

\section{Staging}

Patients were staged based on modified BCLC staging and treatment strategies determined for stages A-D [8-10]. Patients without information on Child-Pugh score $(n=169)$, lymph node status ( $n=97)$, metastatic status $(n=8)$, tumor size $(n=44)$, or bilirubin level $(n=5)$ were classified as "unstaged." Performance status was not available for use in classification. Patients were classified as stage 0 if they had a Child-Pugh score of A, a single tumor of $<2 \mathrm{~cm}$, and a bilirubin of $<2 \mathrm{mg} / \mathrm{dl}$. Patients were categorized as stage A if they had a Child-Pugh score of A-B and a single tumor or three nodules of $<3 \mathrm{~cm}$. Patients with a Child-Pugh score of A-B with multinodular tumors were classified as stage B. Patients classified as stage 0-B could have no portal invasion, nodal involvement, or metastatic disease. Stage $C$ included patients with a ChildPugh score of A-B with portal invasion, nodal involvement, or metastatic disease. Finally, patients with a Child-Pugh score of $\mathrm{C}$ were classified as stage D.

\section{Treatment Definition}

For stage 0 patients or stage A patients who had a single nodule and a bilirubin of $<2 \mathrm{mg} / \mathrm{dL}$, liver resection was recommended [10]. For patients with stage $A$ and three nodules of $<3 \mathrm{~cm}$ a liver transplant or radiofrequency ablation was advised. Treatment for patients with stage B was chemoembolization. "New agents" were recommended therapy for stage C, and best supportive care for patients with stage D. Treatments were defined as therapies planned or administered prior to progression or recurrence of the disease.

\section{Analysis}

Bivariate analyses were performed to characterize the association between stage and selected variables, including demographic characteristics, clinical information, and treatment. Data are presented as row percentages by stage and as column percentages for the entire sample. Multivariable logistic regression analyses were used to examine factors hypothesized to be associated with receipt of specific treatments (surgery, transplant, embolization, and Sorafenib) for all patients as well as a smaller number of factors thought to be associated with appropriate therapy stratified by stage at diagnosis. Because of the limited number of patients $(n=15)$, stage-specific analyses were not conducted for stage 0 . We used multivariable Cox proportional hazard models to examine all-cause mortality with follow-up through December 2009, while simultaneously adjusting for age, race/ethnicity, insurance status, residency training program, stage, Charlson Score, income, and stage-appropriate therapy. All tests for significance were two-sided and considered significant at $\mathrm{p}<0.05$.

All estimates were weighted using sample weights, calculated as the inverse of the sampling proportion for each sampling stratum, to obtain estimates that are representative of all eligible patients from which the sample was drawn. We used the statistical software SAS (SAS Institute, Cary, NC, USA) and SUDAAN (RTI International, Research Triangle Park, NC, USA); SUDAAN allows for sample weights and adjusts the standard errors appropriately.

\section{Results}

The majority of staged patients were classified as stage B (n=268) (table 1), although a large number of patients were unstaged $(n=323)$, primarily due to missing Child-Pugh scores or lymph node status. Patients aged 80 or older, Hispanics, and patients treated in hospitals with no residency training program were more likely to be unstaged. The majority of patients 
with stage 0 and A HCC were alive at the end of 2009 (>70\%); while only $11 \%$ and $16 \%$ of stage $\mathrm{C}$ and $\mathrm{D}$ patients, respectively, were alive.

\section{Surgery}

Treatments were associated with stage of disease (table 2). Surgery as the only cancer therapy was most common for patients with stage B disease, $26.8 \%$, followed by patients with stage $0,12.4 \%$. Patients diagnosed with stage 0 or B were also most likely to receive multiple therapies, $35.1 \%$ and $34.2 \%$, respectively. In a multivariable analysis, younger patients, those treated in hospitals with a residency training program, and those diagnosed at earlier stages were significantly more likely to receive surgery, while non-Hispanic black patients and Medicaid patients were significantly less likely to receive surgery (table 3).

\section{Transplants}

Transplants were more frequent in earlier stage disease (36.5\% of stage 0 and $48.1 \%$ of stage A (table 2). The multivariable analysis of transplants was limited to patients with insurance and stages A through unstaged. Patients aged 50 or older, non-Hispanic black and Hispanic patients, Medicaid patients, treatment in a hospital with no residency training program, stage B-D and unstaged disease, and those with a Charlson score of 0 were significantly less likely to receive a transplant (table 3). Among stage A patients for whom liver transplant, tumor embolization, or radiofrequency ablation (RFA) were recommended treatments, non-Hispanic black and Hispanic patients, patients with the highest quartile of income, and patients with Medicaid were significantly less likely to receive these therapies (table 4).

\section{Tumor Embolization}

Tumor embolization without other therapy was infrequent (table 2). It was most often performed in patients with stage B disease in combination with systemic chemotherapy $(15.3 \%)$ (data not shown). In the multivariable analysis, being in the second quartile of income (less likely to undergo embolization) was the only factor associated with embolization (table 3). Tumor embolization for patients with stage B disease was performed significantly less often for patients in the second quartile of income and patients with no reported cirrhosis (table 4).

\section{Systemic Therapy}

Sorafenib as a single therapy was prescribed most often to stage $\mathrm{C}$ patients $(13.6 \%$, table 2 ), but was also administered to $12.3 \%$ of stage 0 patients. Sunitinib and interferon were received by less than $1 \%$ of patients irrespective of stage (data not shown). In a multivariable analysis, sorafenib use was significantly higher in Asian/Pacific Islanders, those in the highest quartile of income and patients with stage $C$ or unstaged disease and significantly lower in patients with no insurance (table 3 ). In the multivariable analysis of stage $C$ patients, those with cirrhosis received sorafenib significantly less often (table 4).

\section{Radiofrequency Ablation}

RFA was used most often for patients with stages 0 and A HCC (table 2). RFA was used alone for $3.7 \%$ of stage 0 patients and $3.9 \%$ of patients with stage $A$.

\section{No Treatment}

Overall, $38.5 \%$ of patients received no therapy, including about half of the stage C, D, and unstaged patients (table 2). In multivariable analysis, age $>70$, the second quartile of 
Table 1. Demographic and hospital characteristics of patients diagnosed with histologically confirmed hepatocellular carcinoma, 2007 (total $n=946$ )

\begin{tabular}{|c|c|c|c|c|c|c|c|c|}
\hline & & \multicolumn{7}{|c|}{ BCLC stage } \\
\hline & & 0 & A & B & $\mathrm{C}$ & $\mathrm{D}$ & Unstaged & Total \\
\hline & & $\begin{array}{l}\mathrm{n}=15 \\
\text { Row \% }\end{array}$ & $\begin{array}{l}n=68 \\
\text { Row } \%\end{array}$ & $\begin{array}{l}\mathrm{n}=268 \\
\text { Row } \%\end{array}$ & $\begin{array}{l}n=155 \\
\text { Row\% }\end{array}$ & $\begin{array}{l}\mathrm{n}=117 \\
\text { Row } \%\end{array}$ & $\begin{array}{l}\mathrm{n}=323 \\
\text { Row } \%\end{array}$ & $\begin{array}{l}\mathrm{n}=946 \\
\text { Column \% }\end{array}$ \\
\hline \multirow[t]{5}{*}{ Age } & $<50$ & 0.4 & 5.8 & 38.6 & 15 & 24.6 & 15.6 & 10.7 \\
\hline & $50-59$ & 1.6 & 9.3 & 16.6 & 19 & 12.2 & 41.4 & 33.8 \\
\hline & $60-69$ & 1.4 & 9.9 & 24.5 & 17.6 & 17 & 29.6 & 26 \\
\hline & $70-79$ & 2.1 & 6.1 & 40.2 & 15 & 10.1 & 26.4 & 19.4 \\
\hline & $80+$ & 0 & 2.4 & 35.5 & 10.4 & 4.9 & 46.7 & 10 \\
\hline \multirow[t]{2}{*}{ Sex } & Male & 1 & 6.8 & 27.8 & 16.9 & 14.5 & 33 & 77.2 \\
\hline & Female & 2.5 & 10.9 & 26.6 & 15.5 & 10.8 & 33.8 & 22.8 \\
\hline \multirow[t]{5}{*}{ Race/ethnicity } & NH white & 1.3 & 9.1 & 25.4 & 17.4 & 15.2 & 31.6 & 48.6 \\
\hline & NH black & 0.4 & 2.9 & 28 & 23.3 & 15.8 & 29.7 & 12.4 \\
\hline & Hispanic & 0 & 7.6 & 23.8 & 13.4 & 14.3 & 40.9 & 17.6 \\
\hline & Asian & 3 & 7.9 & 36.3 & 11.9 & 8.2 & 32.6 & 20.5 \\
\hline & AI/AN & 0 & 4.3 & 8.7 & 47.8 & 8.7 & 30.4 & 0.9 \\
\hline \multirow[t]{2}{*}{ Marital status } & $\begin{array}{l}\text { Married/ } \\
\text { living as a married }\end{array}$ & 1.7 & 9.4 & 30.1 & 16.9 & 14.3 & 27.5 & 54.9 \\
\hline & Other & 0.8 & 5.8 & 24.4 & 16.1 & 12.7 & 40.1 & 45.1 \\
\hline \multirow[t]{5}{*}{ Insurance status } & Private & 1.6 & 7.9 & 29.9 & 16.5 & 12.4 & 31.7 & 60.1 \\
\hline & Any Medicaid & 0.3 & 10.1 & 23.6 & 11.8 & 21.3 & 32.9 & 24.5 \\
\hline & Medicare only & 3.2 & 5.8 & 26.9 & 22.6 & 7.2 & 34.3 & 9.9 \\
\hline & No insurance & 0 & 0 & 21.1 & 30.8 & 4.3 & 43.7 & 4.7 \\
\hline & Unknown & 0 & 0 & 11.5 & 9.2 & 4.6 & 74.8 & 0.8 \\
\hline \multirow[t]{2}{*}{ Residency program } & Yes & 1.8 & 10.3 & 30.5 & 16.9 & 14.7 & 25.7 & 64.3 \\
\hline & No/Unknown & 0.5 & 3.2 & 22.1 & 15.9 & 11.8 & 46.6 & 35.7 \\
\hline \multirow[t]{4}{*}{ Income (quartiles) } & $<\$ 37,820$ & 0.8 & 2.3 & 24.8 & 16.6 & 18.7 & 36.8 & 24.9 \\
\hline & $\$ 37,820-\$ 50,033$ & 0.8 & 9.6 & 23.1 & 22.9 & 8.2 & 35.5 & 24.9 \\
\hline & $\$ 50,034-\$ 67,103$ & 1.9 & 9.2 & 27.9 & 13.8 & 15.6 & 31.7 & 25.1 \\
\hline & $\$ 67,104+$ & 1.9 & 10 & 34.3 & 13 & 12 & 28.8 & 25.1 \\
\hline $\begin{array}{l}\text { Vital status } \\
\text { (column\%) } 2009\end{array}$ & Alive & 71 & 71.9 & 37.8 & 11.1 & 15.8 & 20.5 & 27.7 \\
\hline
\end{tabular}

$\mathrm{NH}=$ non-Hispanic; $\mathrm{AI} / \mathrm{AN}=$ American Indians/Alaskan Natives.

income, Medicaid, no insurance, being treated in a facility with no residency training program, and later stage of disease was associated with a lack of cancer therapy (table 3). In multivariable analysis, the use of best supportive therapy for stage D patients, although not likely curative, was not associated with race/ethnicity, income, or cirrhosis (table 4). However, among these patients, those with Medicaid were less likely to receive any cancer therapy. 
Table 2. Clinical characteristics of patients diagnosed with histologically confirmed hepatocellular carcinoma, 2007 weighted percentages

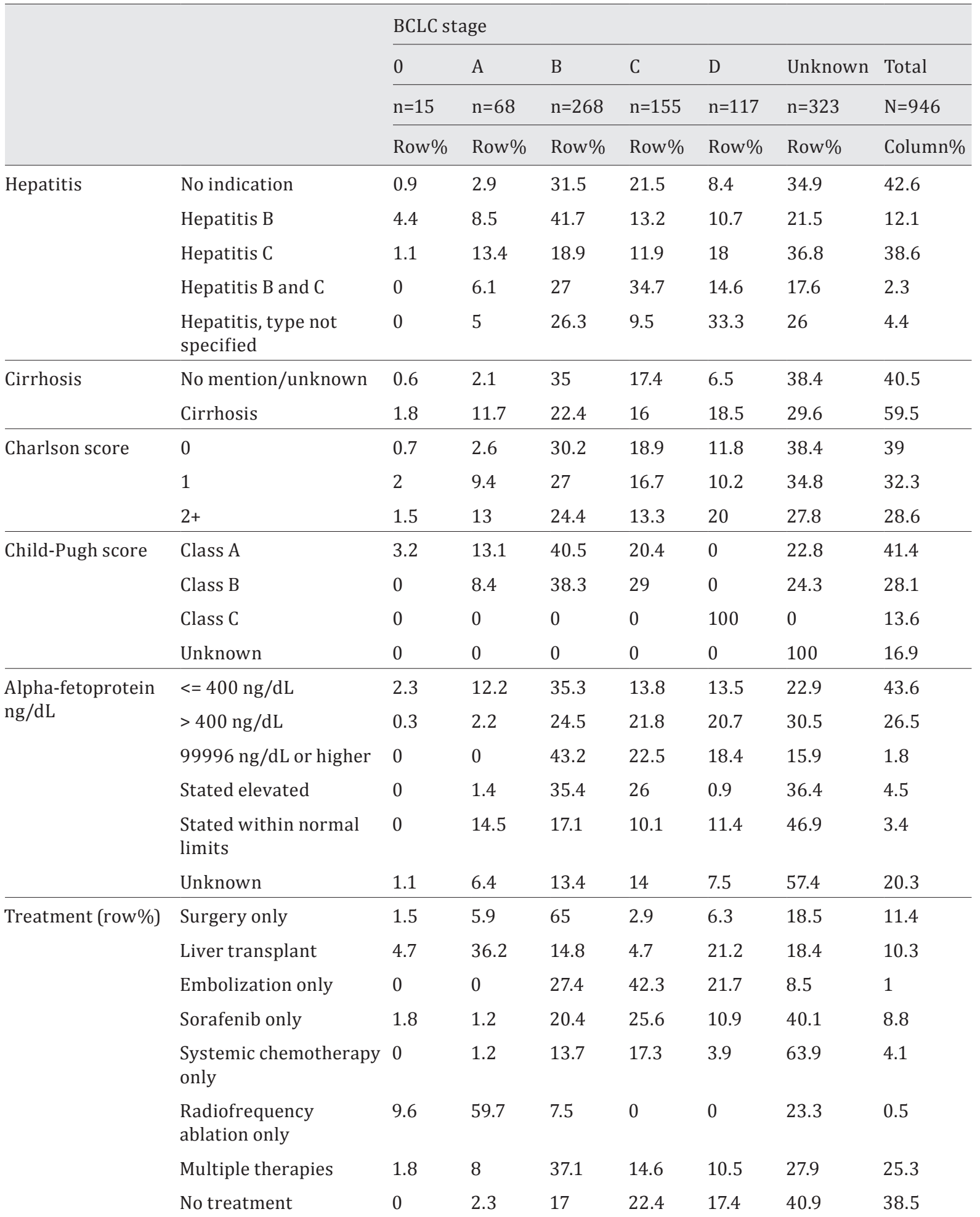


Table 2. (continue) Clinical characteristics of patients diagnosed with histologically confirmed hepatocellular carcinoma, 2007 weighted percentages

\begin{tabular}{|c|c|c|c|c|c|c|c|c|}
\hline & & \multicolumn{7}{|c|}{ BCLC stage } \\
\hline & & 0 & A & B & $\mathrm{C}$ & $\mathrm{D}$ & Unknown & Total \\
\hline & & $\mathrm{n}=15$ & $\mathrm{n}=68$ & $n=268$ & $n=155$ & $n=117$ & $n=323$ & $\mathrm{~N}=946$ \\
\hline & & Row\% & Row\% & Row\% & Row\% & Row\% & Row\% & Column $\%$ \\
\hline \multirow{8}{*}{$\begin{array}{l}\text { Treatment } \\
\text { (column\%) }\end{array}$} & Surgery only & 12.4 & 8.6 & 26.8 & 2 & 5.3 & 6.3 & 11.4 \\
\hline & Liver transplant & 36.5 & 48.1 & 5.5 & 2.9 & 16 & 5.7 & 10.3 \\
\hline & Embolization only & 0 & 0 & 1 & 2.7 & 1.7 & 0.3 & 1 \\
\hline & Sorafenib only & 12.3 & 1.4 & 6.5 & 13.6 & 7.1 & 10.7 & 8.8 \\
\hline & $\begin{array}{l}\text { Systemic chemotherapy } \\
\text { only }\end{array}$ & 0 & 0.6 & 2 & 4.3 & 1.2 & 7.9 & 4.1 \\
\hline & $\begin{array}{l}\text { Radiofrequency } \\
\text { ablation only }\end{array}$ & 3.7 & 3.9 & 0.1 & 0 & 0 & 0.4 & 0.5 \\
\hline & Multiple therapies & 35.1 & 26.2 & 34.2 & 22.4 & 19.6 & 21.3 & 25.3 \\
\hline & No treatment & 0 & 11.2 & 23.7 & 52.1 & 49.2 & 47.5 & 38.5 \\
\hline $\begin{array}{l}\text { Appropriate therapy } \\
\text { (column\%) }\end{array}$ & & 84 & 59.5 & 31.7 & 26.5 & $50.8^{*}$ & $52.5^{*}$ & 43.2 \\
\hline
\end{tabular}

*Any therapy was considered appropriate.

\section{Survival}

Increased all-cause mortality was associated with being aged 50 or older, treatment in a hospital lacking a residency training program, stage B-D and unstaged patients, and patients who failed to receive appropriate therapy (table 5). These results were similar when the analysis was restricted to patients with an assigned stage only.

\section{Discussion}

This population-based study of HCC treatment found that a varying set of clinical and demographic factors predicted the type of therapy received depending on patients' stage at diagnosis. Our primary findings were that appropriate therapy for stage A patients was given significantly less often to non-Hispanic blacks, Hispanics, and patients in the highest quartile of income. Patients with stage A or D HCC were less likely to receive appropriate therapy if they had Medicaid. In multivariable analyses of all-cause mortality, younger age, treatment in a hospital with a residency training program, earlier stage at diagnosis, and adequate treatment were associated with improved survival.

Although chemoembolization is the recommended therapy, surgery alone was the therapy of choice for slightly more than one-quarter of patients with stage B HCC. The predictors of surgery for early-stage HCC were similar to those given in a report on Medicare patients [13]. While the guidelines for BCLC stage B therapy do not recommend surgery [14], this remains controversial [15]. A recent report suggested that selected stage B patients may have a better outcome with more aggressive treatment, including surgery [16]. In the current study, after 


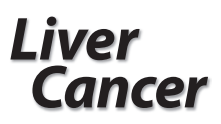

\begin{tabular}{|c|c|}
\hline \multicolumn{2}{|l|}{ Liver Cancer 2015;4:70-83 } \\
\hline $\begin{array}{l}\text { DOI: } 10.1159 / 000367729 \\
\text { Publisned online: February 17, } 2015\end{array}$ & $\begin{array}{l}\text { (c) } 2015 \text { S. Karger AG, Basel } \\
\text { www.karger.com/lic }\end{array}$ \\
\hline
\end{tabular}

Table 3. Multivariable analysis of clinical and non-clinical variables for stage A-D and unstaged patients and selected treatments (not mutually exclusive)

\begin{tabular}{|c|c|c|c|c|c|c|c|c|c|c|c|}
\hline \multirow[t]{2}{*}{$\begin{array}{l}\text { Independent/ } \\
\text { dependent }\end{array}$} & & \multicolumn{2}{|c|}{$\begin{array}{l}\text { Had surgery } \\
(n=910)\end{array}$} & \multicolumn{2}{|c|}{$\begin{array}{l}\text { Had transplant } \\
(n=877)\end{array}$} & \multicolumn{2}{|c|}{$\begin{array}{l}\text { Embolization }^{a} \\
(n=910)\end{array}$} & \multicolumn{2}{|c|}{$\begin{array}{l}\text { Sorafenib } \\
(n=910)\end{array}$} & \multicolumn{2}{|c|}{$\begin{array}{l}\text { No treatment } \\
(n=910)\end{array}$} \\
\hline & & OR & $(95 \% \mathrm{CI})$ & OR & $(95 \% \mathrm{CI})$ & OR & $(95 \% \mathrm{CI})$ & OR & $(95 \% \mathrm{CI})$ & OR & $(95 \% \mathrm{CI})$ \\
\hline \multirow[t]{4}{*}{ Age at diagnosis } & $<50$ & 1.0 & & 1.0 & & 1.0 & & 1.0 & & 1.0 & \\
\hline & $50-59$ & 0.4 & $(0.2,0.7)$ & 0.3 & $(0.1,0.7)$ & 0.6 & $(0.3,1.4)$ & 2.6 & $(0.9,7.5)$ & 1.4 & $(0.6,3.2)$ \\
\hline & $60-69$ & 0.4 & $(0.2,0.95)$ & 0.2 & $(0.1,0.5)$ & 1 & $(0.4,2.6)$ & 1.6 & $(0.6,4.8)$ & 1.5 & $(0.6,3.6)$ \\
\hline & $70+$ & 0.1 & $(0.1,0.3)$ & 0.01 & $(0,0.03)$ & 0.5 & $(0.2,1.2)$ & 1.3 & $(0.4,3.8)$ & 3.8 & $(1.8,8.0)$ \\
\hline \multirow[t]{4}{*}{ Race/ethnicity } & NH white & 1.0 & & 1.0 & & 1.0 & & 1.0 & & 1.0 & \\
\hline & NH black & 0.3 & $(0.2,0.7)$ & 0.4 & $(0.1,0.9)$ & 0.7 & $(0.3,1.4)$ & 1.5 & $(0.8,2.9)$ & 1.4 & $(0.7,2.8)$ \\
\hline & Hispanic & 0.6 & $(0.3,1.4)$ & 0.3 & $(0.1,0.7)$ & 0.7 & $(0.3,1.4)$ & 1.6 & $(0.8,3.2)$ & 0.9 & $(0.5,1.8)$ \\
\hline & Asian/PI & 0.9 & $(0.5,1.6)$ & 0.8 & $(0.4,1.7)$ & 0.6 & $(0.4,1.2)$ & 2.1 & $(1.1,3.8)$ & 0.7 & $(0.4,1.3)$ \\
\hline \multirow{4}{*}{$\begin{array}{l}\text { Income } \\
\text { (quartiles) }\end{array}$} & $<\$ 37,820$ & 1.0 & & 1.0 & & 1.0 & & 1.0 & & 1.0 & \\
\hline & $\begin{array}{l}\$ 37,820- \\
50,033\end{array}$ & 1.0 & $(0.5,1.9)$ & 0.8 & $(0.3,2.6)$ & 0.4 & $(0.2,0.9)$ & 0.8 & $(0.4,1.8)$ & 1.8 & $(1.03,3.1)$ \\
\hline & $\begin{array}{l}\$ 50,034- \\
67,103\end{array}$ & 2.0 & $(0.9,4.7)$ & 1.9 & $(0.6,5.6)$ & 0.9 & $(0.4,2.2)$ & 0.6 & $(0.3,1.4)$ & 1 & $(0.5,2.1)$ \\
\hline & $\$ 67,104+$ & 0.7 & $(0.3,1.4)$ & 0.3 & $(0.1,1.4)$ & 0.9 & $(0.4,2.1)$ & 2.2 & $(1.2,3.9)$ & 0.6 & $(0.3,1.3)$ \\
\hline \multirow[t]{4}{*}{ Insurance } & Private & 1.0 & & 1.0 & & 1.0 & & 1.0 & & 1.0 & \\
\hline & $\begin{array}{l}\text { Any } \\
\text { Medicaid }\end{array}$ & 0.6 & $(0.3,0.98)$ & 0.4 & $(0.2,0.98)$ & 0.6 & $(0.3,1.06)$ & 0.8 & $(0.4,1.7)$ & 2.1 & $(1.3,3.5)$ \\
\hline & $\begin{array}{l}\text { Medicare } \\
\text { only }\end{array}$ & 0.9 & $(0.4,1.8)$ & 1.9 & $(0.5,7.0)$ & 0.7 & $(0.3,1.5)$ & 1.2 & $(0.5,2.9)$ & 1.1 & $(0.5,2.2)$ \\
\hline & $\begin{array}{l}\text { No } \\
\text { insurance }\end{array}$ & 0.3 & $(0.1,1.1)$ & No pa & atients & 0.7 & $(0.2,2.4)$ & 0.1 & $(0.04,0.5)$ & 5.5 & $(2.1,14.4)$ \\
\hline \multirow{2}{*}{$\begin{array}{l}\text { Residency } \\
\text { training }\end{array}$} & Yes & 1.0 & & 1.0 & & 1.0 & & 1.0 & & 1.0 & \\
\hline & $\begin{array}{l}\text { No/ } \\
\text { Unknown }\end{array}$ & 0.4 & $(0.2,0.8)$ & 0.1 & $(0.04,0.5)$ & 0.7 & $(0.4,1.3)$ & 0.9 & $(0.5,1.8)$ & 2.4 & $(1.5,4.0)$ \\
\hline \multirow[t]{5}{*}{ Stage } & $\mathrm{A}$ & 1.0 & & 1.0 & & 1.0 & & 1.0 & & 1.0 & \\
\hline & B & 0.5 & $(0.2,1.2)$ & 0.06 & $(0.02,0.2)$ & 1.3 & $(0.5,3.23)$ & 2.7 & $(0.8,9.4)$ & 2 & $(0.6,6.0)$ \\
\hline & $\mathrm{C}$ & 0.1 & $(0,0.2)$ & 0.03 & $(0.01,0.2)$ & 0.6 & $(0.2,1.6)$ & 8.8 & $(2.5,31.4)$ & 7.2 & $(2.2,24.1)$ \\
\hline & $\mathrm{D}$ & 0.2 & $(0.1,0.6)$ & 0.1 & $(0.1,0.3)$ & 0.6 & $(0.2,1.9)$ & 3.4 & $(0.9,12.9)$ & 8.3 & $(2.4,28.5)$ \\
\hline & Unstaged & 0.2 & $(0.1,0.5)$ & 0.1 & $(0.04,0.3)$ & 0.8 & $(0.3,2.3)$ & 4.2 & $(1.3,13.6)$ & 5 & $(1.6,15.6)$ \\
\hline \multirow{2}{*}{$\begin{array}{l}\text { Charlson } \\
\text { score }\end{array}$} & 0 & 1.0 & & 1.0 & & 1.0 & & 1.0 & & 1.0 & \\
\hline & $1+$ & 0.7 & $(0.4,1.2)$ & 3.2 & $(1.5,6.7)$ & 1.3 & $(0.7,2.5)$ & 1.2 & $(0.7,2.1)$ & 0.8 & $(0.5,1.5)$ \\
\hline
\end{tabular}

Bold odds ratio/95\% confidence interval [OR (95\% CI)] significant at $\mathrm{p}<0.05$. Asian $/ \mathrm{PI}=$ Asian/Pacific Islanders. ${ }^{a}$ Any method of embolization. 
Table 4. Multivariable analysis by stage of disease at diagnosis and appropriate therapy

\begin{tabular}{|c|c|c|c|c|c|c|c|}
\hline & & $\begin{array}{l}\text { Stage A only } \\
(\mathrm{n}=67) \\
\text { Liver transplant } \\
\text { or tumor } \\
\text { embolization or } \\
\text { RFA }\end{array}$ & \multicolumn{2}{|c|}{$\begin{array}{l}\text { Stage B only } \\
(\mathrm{n}=265) \\
\text { Tumor emboliza- } \\
\text { tion }\end{array}$} & \multicolumn{2}{|c|}{$\begin{array}{l}\text { Stage C only } \\
(\mathrm{n}=150) \\
\text { Sorafenib vs. no } \\
\text { sorafenib }\end{array}$} & $\begin{array}{l}\text { Stage D only } \\
(\mathrm{n}=110) \\
\text { Any therapy } \\
\text { vs. none }\end{array}$ \\
\hline & & OR $\quad(95 \% \mathrm{CI})$ & OR & $(95 \% \mathrm{CI})$ & OR & $(95 \% \mathrm{CI})$ & OR $(95 \% \mathrm{CI})$ \\
\hline \multirow[t]{4}{*}{ Race/ethnicity } & NH white & 1.0 & 1.0 & & 1.0 & & 1.0 \\
\hline & NH black & $0.1(0.01,0.96)$ & 1.8 & $(0.6,6.0)$ & 1.0 & $(0.3,3.9)$ & $0.7 \quad(0.2,2.6)$ \\
\hline & Hispanic & $0.1(0.01,0.5)$ & 1.0 & $(0.3,3.3)$ & 1.5 & $(0.4,6.0)$ & $0.3(0.1,1.3)$ \\
\hline & $\begin{array}{l}\text { Asian/Pacific } \\
\text { Islanders }\end{array}$ & $0.5(0.1,2.0)$ & 0.8 & $(0.3,2.0)$ & 1.3 & $(0.3,5.5)$ & $0.7(0.2,3.3)$ \\
\hline \multirow[t]{4}{*}{ Income (quartiles) } & $<\$ 37,820$ & 1.0 & 1.0 & & & & 1.0 \\
\hline & $\$ 37,820-50,033$ & $0.4 \quad(0.03,4.5)$ & 0.3 & $(0.1,0.8)$ & 1.5 & $(0.3,6.6)$ & $0.2(0.1,1.04)$ \\
\hline & $\$ 50,034-67,103$ & $0.6(0.05,7.2)$ & 1.0 & $(0.4,3.1)$ & 1.8 & $(0.3,9.8)$ & $0.4 \quad(0.1,1.6)$ \\
\hline & $\$ 67,104+$ & $0.1(0.0,0.9)$ & 1.0 & $(0.4,2.9)$ & 3.0 & $(0.7,13.4)$ & $1.6(0.4,6.7)$ \\
\hline \multirow[t]{4}{*}{ Insurance } & Private & 1.0 & 1.0 & & & & 1.0 \\
\hline & Any Medicaid & $0.1(0.03,0.6)$ & 0.5 & $(0.2,1.3)$ & 0.9 & $(0.2,3.9)$ & $0.2(0.1,0.6)$ \\
\hline & Medicare only & $0.2(0.02,1.6)$ & 0.8 & $(0.3,2.5)$ & 0.9 & $(0.2,3.7)$ & $1.2(0.2,9.4)$ \\
\hline & No insurance & No patients & 0.1 & $(0.01,1.4)$ & 0.2 & $(0.03,1.6)$ & \\
\hline \multirow[t]{2}{*}{ Cirrhosis } & No/unknown & & 1.0 & & & & 1.0 \\
\hline & Yes & & 2.6 & $(1.3,5.4)$ & 0.3 & $(0.1,0.9)$ & $1.5(0.4,5.0)$ \\
\hline \multirow{2}{*}{$\begin{array}{l}\text { Charlson } \\
\text { score }\end{array}$} & 0 & 1.0 & & & & & \\
\hline & $1+$ & $2.6(0.3,24.3)$ & & & & & \\
\hline
\end{tabular}

Bold odds ratio/95\% confidence interval [OR (95\% CI)] significant at $\mathrm{p}<0.05$.

adjusting for other factors, receipt of surgery was not significantly different for patients with stage B compared to patients with stage A disease.

Among patients with stage A disease, those in the highest quartile of income were less likely to undergo RFA, tumor embolization, or transplantation. It is possible that these patients had better liver function and were therefore candidates for surgical resection alone. Overall, among patients with stage A disease, $8.6 \%$ underwent surgical resection only, while $48 \%$ received a liver transplant. In fact, in the current study, a liver transplant was used 10 times as often in patients with cirrhosis $(16.3 \%)$ compared to patients with no reported cirrhosis (1.5\%), suggesting patients with better liver function were more likely to have a resection. Furthermore, when patients of similar stage were compared, those with no reported cirrhosis more often underwent surgery than patients with cirrhosis. Although, patients with a Charlson score of 0 were less likely to receive a transplant, it is possible that patients with fewer serious comorbid conditions also had better liver function and therefore had a liver resection or other less aggressive therapy. This may also be a result of more detailed recording of comorbidities in the medical record of patients about to undergo a liver transplant. Controversy remains regarding the use of surgery or a transplant. A study of patients with "preserved liver function" reported a longer survival among patients with surgically 
Table 5. Cox proportional hazard model - association between clinical and non-clinical factors and all-cause mortality (hazard ratios, 95\% confidence intervals)

\begin{tabular}{|c|c|c|c|c|}
\hline \multirow[t]{4}{*}{ Age group } & $<50$ & 1.0 & & \\
\hline & $50-59$ & 2.1 & 1.2 & 3.5 \\
\hline & $60-69$ & 2.1 & 1.2 & 3.6 \\
\hline & $70+$ & 1.8 & 1.1 & 3.1 \\
\hline \multirow[t]{4}{*}{ Race/ethnicity } & NH white & 1.0 & & \\
\hline & NH black & 1.0 & 0.8 & 1.4 \\
\hline & Hispanic & 1.2 & 0.9 & 1.6 \\
\hline & Asian & 0.8 & 0.6 & 1.2 \\
\hline \multirow[t]{4}{*}{ Insurance status } & Private & 1.0 & & \\
\hline & Any Medicaid & 1.2 & 0.95 & 1.5 \\
\hline & Medicare only & 1.0 & 0.8 & 1.4 \\
\hline & No insurance & 0.9 & 0.5 & 1.7 \\
\hline \multirow[t]{2}{*}{ Residency program } & Yes & 1.0 & & \\
\hline & No/Unk & 1.4 & 1.1 & 1.9 \\
\hline \multirow[t]{6}{*}{ Stage } & 0 & 1.6 & 0.6 & 4.3 \\
\hline & A & 1.0 & & \\
\hline & B & 2.8 & 1.5 & 5.0 \\
\hline & $\mathrm{C}$ & 6.8 & 3.7 & 12.5 \\
\hline & $\mathrm{D}$ & 10.6 & 5.7 & 19.5 \\
\hline & Unstaged & 5.4 & 3.0 & 9.8 \\
\hline \multirow[t]{2}{*}{ Charlson score } & 0 & 1.0 & & \\
\hline & $1+$ & 0.9 & 0.7 & 1.2 \\
\hline \multirow[t]{4}{*}{ Income quartiles } & $<\$ 37,820$ & 1.0 & & \\
\hline & $\$ 37,820-50,033$ & 1.1 & 0.8 & 1.5 \\
\hline & $\$ 50,034-67,103$ & 0.8 & 0.5 & 1.1 \\
\hline & $\$ 67,104+$ & 1.2 & 0.9 & 1.6 \\
\hline \multirow[t]{2}{*}{ Therapy } & Appropriate therapy & 1.0 & & \\
\hline & No & 2.4 & 1.9 & 3.2 \\
\hline
\end{tabular}

Bold hazard ratios and 95\% confidence intervals significant at $\mathrm{p}<0.05$.

resected HCC compared with those receiving a liver transplant [17]. However, another study reported that patients who received a liver transplant despite exceeding the Milan Criteria experienced similar overall survival compared to liver resection patients [18].

Tumor embolization accounted for a substantial percentage of treatment, most often in combination with other therapies. A meta-analysis suggested that chemoembolization improved survival in patients with unresectable disease [19], although a more recent metaanalysis suggested that survival benefits were not clear [20,21]. In 2006, Sangro et al. concluded that radioembolization could be safely performed even for patients with cirrhosis [22]. Overall survival was poorer for nonsurgical therapies compared to liver resection in a study of 
non-cirrhotic HCC patients after adjusting for demographic factors, risk factors, and tumor characteristics [17]. Our data indicate that tumor embolization alone or in combination with other treatments was undergone by $29.6 \%$ patients with cirrhosis of the liver compared to only $14.6 \%$ in those with no reported cirrhosis. In a 2012 review, the use of sorafenib with transcatheter arterial chemoembolization reportedly improved time to progression [23]. These data suggest that in 2007 sorafenib was infrequently given with tumor embolization, i.e., to $3 \%$ of patients with stage B and $6.3 \%$ of patients with stage C.

The current recommendation for patients diagnosed with advanced disease is sorafenib. In 2007 the recommendation was for a clinical trial or "new agents." Initially approved in 2005 for kidney cancer, sorafenib was subsequently approved for unresectable HCC in November 2007. In a placebo controlled clinical trial, sorafenib showed a significant survival advantage [24]. Even though not approved until November 2007, the majority of sorafenib included in this study was given prior to that date. Notably, in this study, sorafenib was used less often for patients with no insurance and more often for those with the highest income. Cost is likely a factor since a month's supply is more than $\$ 10,000$ [25].

The BCLC staging and treatment strategy developed by Llovet et al. suggests that RFA should be given for early-stage disease [9]. However, a recent meta-analysis of studies reported between 2000 and 2010 found that RFA was a valuable treatment also in advanced disease [26]. In our study, we found that RFA was most often used in stage 0 patients, but fewer than $3 \%$ of stage $C$, D, or unstaged patients received RFA, suggesting that physicians were limiting the use of RFA to early-stage disease.

For patients with late-stage disease, therapy does not provide a cure. In this population-based sample, about half of patients with stage $\mathrm{C}, \mathrm{D}$, and unstaged disease received no cancer-directed therapy. Although potentially curable, $11 \%$ and $24 \%$ of patients with stage A and B disease, respectively, did not receive cancer therapy. Generally, these patients were over age 80 and had Medicaid or no insurance. While not directly comparable, an analysis of SEER-Medicare HCC patients found that curative therapy was not provided for the majority of the patients and, of patients with favorable tumor characteristics, only one-third received such therapy [27]. Our population-based sample that also includes individuals under the age of 65 found that older patients were less likely to receive surgery and, appropriately, no patient aged 80 or older received a transplant. Additionally, among stage D patients, lack of insurance was associated with lack of therapy.

Mortality was significantly related to stage of disease, age, treatment in a hospital with an approved residency training program, and the use of stage-appropriate therapy. Our study and those of others demonstrates that survival is significantly better for patients diagnosed with early-stage disease $[28,29]$. A recent study found an increase in the diagnosis of smaller HCC [30]. This early detection is likely a result of improved surveillance for patients with risk factors such as hepatitis and cirrhosis [31]. However, the United State Preventive Services Task Force has no recommendation for routine screening for HCC or cirrhosis and reportedly did not find sufficient evidence of improved HCC outcome related screening for hepatitis B or C [32]. Continued efforts to promote early detection in the community setting should be encouraged through education of health care providers who will serve as the first line of interaction with these individuals.

The association between the use of appropriate therapy and survival is provocative. However, this was not a randomized trial and it may be that patients who failed to receive adequate therapy were poor candidates for these treatments. Mortality in the current study increased with age even after adjusting for other factors, including adequate therapy. Findings from a study of 36 patients aged 80 or older compared to 668 younger patients conducted in Japan suggested that advanced stage, but not age, was associated with poorer survival [33]. Although patients treated in hospitals with a residency training program were less likely 
to die, these hospitals also performed more surgery and transplants. This may suggest that patients with very poor prognoses or a preference for non-aggressive therapy did not seek care in these facilities. While our study focuses on HCC cases diagnosed in 2007 only, future studies should examine continued adoption of new therapies over time and risk factors for inadequate therapy.

\section{Strengths and Limitations}

We acknowledge the following potential limitations. Although comorbidities were collected and the Charlson score or cirrhosis was used in the analyses, performance status was not available in the current study. Patient and physician preferences for treatments were unknown. However, therapy decreased with increasing age, suggesting that there may have been a preference by the patient or physician for less aggressive therapy due to age, frailty, or lack of social support. Not all patients in the study could be assigned a stage. This group was likely heterogeneous with some cases truly not staged, while others were assigned to this category because information was unavailable in their medical records. We do not have information on the management of hepatitis or cirrhosis in these patients or surveillance of patients with these diseases. Both surveillance, as recommended by the American Association for the Study of Liver Disease [14], and active treatment of these risk factors might influence the outcome for these patients. Unfortunately, a recent report noted very poor compliance with these guidelines for patients with hepatitis B followed in a large academic center [34].

Despite these limitations, this study of a population-based sample of nearly 950 HCC patients from diverse geographic regions diagnosed and treated in community-based health care settings reflects HCC management in 2007 and the resulting survival. It also reflects the inadequacy of current therapies for HCC, thus highlighting the need for improved prevention strategies to hopefully decrease the need for the current, mostly non-curative therapies. Knowledge of treatment disparities can inform strategies for promoting appropriate therapy now and as new agents are developed for HCC.

\section{Conflict of Interest}

None reported.

Funding Sources: Supported by National Cancer Institute contracts: HHSN261201000024C; HSN261201000025C; HHSN261201000032C; HHSN261201000027C; HHSN261201000026C; HHSN261201000140C; HHSN261201000037C; HHSN261201000033C; HSN261201000034C; HHSN261201000035C; HHSN261201000029C; HHSN261201000031C; HSN261201000028C; HHSN261201000030C. Dr. Parsons is supported by K07CA175063.

All authors contributed to the conception and design; the acquisition, analysis, or interpretation of the data; drafting of the article; or critical revision for important intellectual content. All authors gave final approval of the version to be published and agreed to be accountable for all aspects of the work in ensuring that questions related to the accuracy or integrity of any part of the article are appropriately investigated and resolved.

This article is a US Government work and, as such, is in the public domain in the United States of America. The content is solely the responsibility of the authors and does not necessarily represent the official views of the National Cancer Institute or the National Institutes of Health

\section{Acknowledgments}

The authors would like to acknowledge the work of the SEER Cancer Registries. This research would not be possible without their efforts. 


\section{References}

1 American Cancer Society: Cancer Facts and Figures 2014. Atlanta, GA: American Cancer Society, 2014. Last accessed June 30, 2014 at http://www.cancer.org/research/cancerfactsstatistics/cancerfactsfigures2014/index.

-2 El-Serag HB, Mason AC: Rising incidence of hepatocellular carcinoma in the United States. N Engl J Med 1999;340:745-750.

-3 Turati F, Talamini R, Pelucchi C, Polesel J, Franceschi S, Crispo, et al: Metabolic syndrome and hepatocellular carcinoma risk. Br J Cancer. 2013 15;108(1):222-228.

4 Mor E, Tur-Kaspa R, Sheiner P, Schwartz M: Treatment of hepatocellular carcinoma associated with cirrhosis in the era of liver transplantation. Ann Intern Med 1998;129:643-653.

5 Adult primary liver cancer treatment PDQ. National Cancer Institute. Last Accessed May 6, 2014 at http:// www.cancer.gov/cancertopics/pdq/treatment/adult-primary-liver/HealthProfessional/page1.

6 NCCN Clinical Practice Guidelines in Oncology: Hepatobiliary Cancers Version 2.2012. National Comprehensive Cancer Center Network 2012. Last accessed March 7, 2012 at http://www.nccn.org/professionals/physician_gls/pdf/hepatobiliary.

-7 Alves RC, Alves D, Guz B, Matos C, Viana M, Harriz M, Terrabuio D, Kondo M, Gampel O, Polletti P: Advanced hepatocellular carcinoma. Review of targeted molecular drugs. Ann Hepatol 2011;10:21-27.

-8 Rahbari NN, Mehrabi A, Mollberg NM, Müller SA, Koch M, Büchler MW, Weitz J: Hepatocellular carcinoma: current management and perspectives for the future. Ann Surg 2011;253:453-469.

-9 Llovet JM: Updated treatment approach to hepatocellular carcinoma. J Gastroenterol 2005;40:225-235.

10 Bruix J, Llovet JM: Major achievements in hepatocellular carcinoma. Lancet 2009;373:614-616.

11 Overview of the SEER Program, National Cancer Institute 2012. Last accessed May 6, 2014 at http://seer. cancer.gov/about/overview.html.

$\$ 12$ Charlson ME, Pompei P, Ales KL, MacKenzie CR: A new method of classifying prognostic comorbidity in longitudinal studies: development and validation. J Chronic Dis 1987;40:373-383.

13 Nathan H, Hyder O, Mayo SC, Hirose K, Wolfgang CL, Choti MA, Pawlik TM: Surgical therapy for early hepatocellular carcinoma in the modern era: a 10-year SEER-Medicare analysis. Ann Surg 2013;258:10221027.

14 Bruix J, Sherman M American Association for the Study of Liver Diseases: Management of hepatocellular carcinoma: an update. Hepatology 2011;53:1020-1022.

-15 Cauchy F, Soubrane 0, Belghiti J: Liver resection for HCC: patient's selection and controversial scenarios. Best Pract Res Clin Gastroenterol 2014;28:881-896.

16 Farinati F, Vanin V, Giacomin A, Pozzan C, Cillo U, Vitale A, Di Nolfo AM, Del Poggio P, Benvegnu' L, Rapaccini G, Zoli M, Borzio F, Giannini EG, Caturelli E, Trevisani F: The Italian Liver Cancer (ITA.LI.CA) group. BCLC stage B hepatocellular carcinoma and transcatheter arterial chemoembolization: a 20-year survey by the Italian Liver Cancer group. Liver Int 2014; doi:10.1111/liv.12649 [Epub ahead of print].

$\checkmark 17$ Koniaris LG, Levi DM, Pedroso FE, Franceschi D, Tzakis AG, Santamaria-Barria JA, Tang J, Anderson M, Misra S, Solomon NL, Jin X, DiPasco PJ, Byrne MM, Zimmers TA: Is surgical resection superior to transplantation in the treatment of hepatocellular carcinoma? Ann Surg 2011;254:527-537, discussion 537538.

18 Canter RJ, Patel SA, Kennedy T, D’Angelica MI, Jarnagin WR, Fong Y, Blumgart LH, Freeman RB, DeMatteo RP, Abt PL: Comparative analysis of outcome in patients with hepatocellular carcinoma exceeding the Milan criteria treated with liver transplantation versus partial hepatectomy. Am J Clin Oncol 2011;34:466471.

19 Llovet JM, Bruix J: Systematic review of randomized trials for unresectable hepatocellular carcinoma: Chemoembolization improves survival. Hepatology 2003;37:429-442.

20 Forner A, Llovet JM, Bruix J: Chemoembolization for intermediate HCC: is there proof of survival benefit? J Hepatol 2012;56:984-986.

21 Wörns MA, Bosslet T, Victor A, Koch S, Hoppe-Lotichius M, Heise M, Hansen T, Pitton MB, Niederle IM, Schuchmann M, Weinmann A, Düber C, Galle PR, Otto G: Prognostic factors and outcomes of patients with hepatocellular carcinoma in non-cirrhotic liver. Scand J Gastroenterol 2012;47:718-728.

-22 Sangro B, Bilbao JI, Boan J, Martinez-Cuesta A, Benito A, Rodriguez J, Panizo A, Gil B, Inarrairaegui M, Herrero I, Quiroga J, Prieto J: Radioembolization using 90Y-resin microspheres for patients with advanced hepatocellular carcinoma. Int J Radiat Oncol Biol Phys 2006;66:792-800.

23 Lencioni R: Chemoembolization for hepatocellular carcinoma. Semin Oncol 2012;39:503-509.

24 Llovet JM, Ricci S, Mazzaferro V, Hilgard P, Gane E, Blanc JF, de Oliveira AC, Santoro A, Raoul JL, Forner A, Schwartz M, Porta C, Zeuzem S, Bolondi L, Greten TF, Galle PR, Seitz JF, Borbath I, Häussinger D, Giannaris T, Shan M, Moscovici M, Voliotis D, Bruix J, SHARP Investigators Study Group: Sorafenib in advanced hepatocellular carcinoma. N Engl J Med 2008;359:378-390.

25 http://www.goodrx.com/nexavar?gclid=CK-P2Nbj87oCFct90godCXQAaw (accessed May, 6, 2014).

-26 Tiong L, Maddern GJ: Systematic review and meta-analysis of survival and disease recurrence after radiofrequency ablation for hepatocellular carcinoma. Br J Surg 2011;98:1210-1224.

$\checkmark 27$ El-Serag HB, Siegel AB, Davila JA, Shaib YH, Cayton-Woody M, McBride R, McGlynn KA: Treatment and outcomes of treating of hepatocellular carcinoma among Medicare recipients in the United States: a population-based study. J Hepatol 2006;44:158-166. 
28 Howlader N, Noone AM, Krapcho M, Garshell J, Miller D, Altekruse SF, Kosary CL, Yu M, Ruhl J, Tatalovich Z, Mariotto A, Lewis DR, Chen HS, Feuer EJ, Cronin KA, editors.: SEER Cancer Statistics Review, 1975-2011, National Cancer Institute. Bethesda, MD, http://seer.cancer.gov/csr/1975_2011/, based on November 2013 SEER data submission, posted to the SEER web site, April 2014. (accessed May 6, 2014).

29 Howlader N, Noone AM, Krapcho M, Neyman N, Aminou R, Altekruse SF, Kosary CL, Ruhl J, Tatalovich Z, Cho H, Mariotto A, Eisner MP, Lewis DR, Chen HS, Feuer EJ, Cronin KA, editors.: SEER Cancer Statistics Review, 1975-2009 (Vintage 2009 Populations), National Cancer Institute. Bethesda, MD, http://seer.cancer.gov/ csr/1975_2009_pops09/, based on November 2011 SEER data submission, posted to the SEER web site, April 2012. (accessed May 6, 2014).

30 Ulahannan SV, Duffy AG, McNeel TS, Kish JK, Dickie LA, Rahma OE, McGlynn KA, Greten TF, Altekruse SF: Earlier presentation and application of curative treatments in hepatocellular carcinoma. Hepatology 2014; [Epub ahead of print].

31 Sherman M: Hepatocellular carcinoma: screening and staging. Clin Liver Dis 2011;15:323-334, vii-X.

32 U.S. Preventive Services Task Force: Hepatitis C Screening. http://www.uspreventiveservicestaskforce. org/uspstf/uspshepc.htm accessed 7/21/2014.

33 Dohmen K, Shirahama M, Shigematsu H, Irie K, Ishibashi H: Optimal treatment strategy for elderly patients with hepatocellular carcinoma. J Gastroenterol Hepatol 2004;19:859-865.

34 Wu Y, Johnson KB, Roccaro G, Lopez J, Zheng H, Muiru A, Ufere N, Rajbhandari R, Kattan O, Chung RT: Poor adherence to AASLD guidelines for chronic hepatitis B management and treatment in a large academic medical center. Am J Gastroenterol 2014;109:867-875. 